\title{
Ensemble fluctuations of the cosmic ray energy spectrum and the intergalactic magnetic field
}

\author{
A. D. Supanitsky \\ Instituto de Astronomía y Física del Espacio (IAFE, CONICET-UBA), \\ CC 67, Suc. 28 (C1428ZAA) Ciudad Autónoma de Buenos Aires, Argentint* \\ G. Medina-Tanco \\ Instituto de Ciencias Nucleares, UNAM, \\ Circuito Exteriror S/N, Ciudad Universitaria, \\ México D. F. 04510, México
}

(Dated: May 8, 2019)

\begin{abstract}
The origin of the most energetic cosmic ray particles is one of the most important open problems in astrophysics. Despite a big experimental effort done in the past years, the sources of these very energetic particles remain unidentified. Therefore, their distribution on the Universe and even their space density are still unknown. It has been shown that different spatial configurations of the sources lead to different energy spectra and composition profiles (in the case of sources injecting heavy nuclei) at Earth. These ensemble fluctuations are more important at the highest energies because only nearby sources, which are necessarily few, can contribute to the flux observed at Earth. This is due to the interaction of the cosmic rays with the low energy photons of the radiation field, present in the intergalactic medium, during propagation. It is believed that the intergalactic medium is permeated by a turbulent magnetic field. Although at present it is still unknown, there are several constraints for its intensity and coherence length obtained from different observational techniques. Charged cosmic rays are affected by the intergalactic magnetic field because of the bending of their trajectories during propagation through the intergalactic medium. In this work, the influence of the intergalactic magnetic field on the ensemble fluctuations is studied. Sources injecting only protons and only iron nuclei are considered. The ensemble fluctuations are studied for different values of the density of sources compatible with the constraints recently obtained from cosmic ray data. Also, the possible detection of the ensemble fluctuations in the context of the future JEM-EUSO mission is discussed.
\end{abstract}

\section{INTRODUCTION}

The origin of the ultrahigh energy cosmic rays (UHECRs) is still unknown. However, big progress on the understanding of this phenomenon has been achieved in past years due to the good quality data taken by current observatories. It is believed that UHECRs are accelerated in extragalactic objects and propagate through the Universe to reach Earth. The main source candidates are active galactic nuclei, radio galaxies, and gamma-ray bursts (see, for instance, Ref. [1]). It is also believed that at low energies the cosmic rays originate in galactic astrophysical objects like supernova remnants [2]. Therefore, the galactic-extragalactic transition of the cosmic ray flux should be located in an intermediate energy region.

The cosmic ray energy spectrum observed at Earth is a very rich source of astrophysical information. It can be roughly approximated by a broken power law with four spectral features: the knee at a few $10^{15} \mathrm{eV}$, the ankle at $\sim 7 \times 10^{18} \mathrm{eV}$, the cutoff or suppression at $\sim 4 \times 10^{19}$ $\mathrm{eV}$, and a second knee at $\sim 10^{17} \mathrm{eV}$, recently reported by the KASCADE-Grande Collaboration [3]. The origin of the suppression is not clear yet. It can be due to the interaction of the cosmic rays with the radiation fields

\footnotetext{
* supanitsky@iafe.uba.ar
}

in the intergalactic medium, on the route to Earth, the inefficiency of the sources to accelerate particles at higher energies, or a combination of both effects.

The composition profile as a function of energy is also a valuable piece of information for the understanding of the origin of the cosmic rays. In this regard, the data collected by The Pierre Auger Observatory, located in the Southern Hemisphere, shows a change that marks the beginning of a transition from light to heavy primaries [4, 5]. Such a transition is not confirmed by the Telescope Array ${ }^{11}$ data, which is more compatible with a proton-dominated composition [6]. However, the present statistics of the Telescope Array data is not enough to distinguish between the composition profile seen by Auger and a proton-dominated case [7]. Note, however, that regarding composition the interpretation of the experimental data is based on shower simulations for which high energy hadronic interaction models, that extrapolate low energy data from accelerators up to the energy of the cosmic rays, are used. Therefore, the composition determination is subject to systematic uncertainties coming from such extrapolation of the high energy hadronic interactions. In any case, there is a real possibility that

\footnotetext{
1 Note that the Telescope Array is located in the Northern Hemisphere.
} 
nuclei heavier than protons dominate the energy spectrum at the highest energies.

At present, there is no identification of any cosmic ray extragalactic source. The data from The Pierre Auger Observatory show an excess in the region of Centaurus A [8]. Also, the data from the Telescope Array observatory show a hot spot located close to the supergalactic plane [9]. However, the statistical significance of both excesses is still quite low, and more data are required to draw any solid conclusion.

The trajectories of the charged cosmic rays are bent by their interaction with the galactic and extragalactic magnetic fields. Heavier nuclei are more affected by these magnetic fields. Therefore, the presence of heavy nuclei at the highest energies would make much more difficult the identification of the sources. The intergalactic magnetic field (IGMF) is poorly known (see Ref. 10] for a review). Faraday rotation measurements show that in the core of galaxy clusters the magnetic field can take values between 1 and $40 \mu \mathrm{G}$. Outside clusters, upper limits to the magnetic field intensity of the parallel component to the line of sight have been obtained by using rotation measurements [11, 12]: $\left\langle B_{\|}^{2} L_{c}\right\rangle^{1 / 2} \lesssim 10 \mathrm{nG} \mathrm{Mpc}{ }^{1 / 2}$, where $L_{c}$ is the coherence length. Recently, a new and very promising technique to constrain the IGMF has been developed. It consists in the study of the effects of a nonnull IGMF on the propagation of gamma rays originated in blazars. For instance, in Ref. [13], it is shown that the application of this new method to the gamma-ray emission coming from the blazar PKS 2155-304, leads to the exclusion of strengths of the IGMF in the range $(0.3-3) \times 10^{-15} \mathrm{G}$ at the $99 \%$ confidence level, under the assumption of a $1 \mathrm{Mpc}$ coherence length. Note that the two methods mentioned above which are used to investigate the IGMF are based on physical principles quite different. While the methods based on gamma-ray observations of blazars make use of the broadening of the angular distribution of photons and the modifications of the energy spectrum at low energies in the case of blazars with hard spectra, the technique based on the observations of Faraday rotation makes use of the rotation of the polarization plane of the polarized light emitted by extragalactic objects.

As mentioned before, the sources of UHECR are not identified yet. Therefore, different spatial configurations of the sources give different energy spectra and, in the case of heavy nuclei at the sources, different composition profiles at Earth. These ensemble fluctuations have been studied in detail in Ref. 14] for the case in which the sources inject either only protons or iron nuclei. Moreover, in those studies the IGMF was neglected. In this work, we study the influence of a non-null IGMF on the ensemble fluctuations. We also consider pure proton and iron for the injected composition, which correspond to the two limiting cases.

The paper is organized as follow. In Sec. II the simulations of the IGMF and the cosmic ray propagation in the intergalactic medium are described. In Sec. III the calcu- lations of the ensemble fluctuations for a non-null IGMF are presented and discussed. The possibility of its observation with future cosmic ray observatories is discussed in Sec. $\nabla$ and we conclude in Sec. [VI.

\section{SIMULATIONS}

As mentioned before the intergalactic magnetic field is poorly known. Therefore, a common approach is to assume that the Universe is filled with a turbulent and homogeneous magnetic field. In this work, the simulation of the field is done following Ref. [15], where an improved version of the method introduced in Ref. [16] is developed. The magnetic field in every point of the space $\mathbf{x}$ is given by

$$
\mathbf{B}(\mathbf{x})=\epsilon \sum_{i=1}^{N} \boldsymbol{\xi}_{i} A\left(k_{i}\right) \cos \left(\mathbf{k}_{i} \cdot \mathbf{x}+\zeta_{i}\right),
$$

where $\epsilon=1 / \sqrt{2}, \zeta_{i}$ is a random phase angle uniformly distributed in $[0,2 \pi]$, and

$$
\mathbf{k}_{i}=k_{i}\left(\begin{array}{c}
\sin \theta_{i} \cos \phi_{i} \\
\sin \theta_{i} \sin \phi_{i} \\
\cos \theta_{i}
\end{array}\right)
$$

with $\theta_{i}$ uniformly distributed in $[0, \pi]$ and $\phi_{i}$ uniformly distributed in $[0,2 \pi]$. The vector $\boldsymbol{\xi}_{i}$ is chosen orthogonal to $\mathbf{k}_{i}$. It can be written as

$$
\boldsymbol{\xi}_{i}=\left(\begin{array}{c}
-\sin \phi_{i} \cos \alpha_{i}+\cos \theta_{i} \cos \phi_{i} \sin \alpha_{i} \\
\cos \phi_{i} \cos \alpha_{i}+\cos \theta_{i} \sin \phi_{i} \sin \alpha_{i} \\
-\sin \theta_{i} \sin \alpha_{i}
\end{array}\right)
$$

where $\alpha_{i}$ is uniformly distributed in $[0,2 \pi]$.

The sum in Eq. (11) extends over $N$ values of the wave number $k=\|\mathbf{k}\|$. The wave numbers $k_{i}$ are separated in such a way that $\log \left(k_{i+1} / k_{i}\right)=\Delta$, where $\Delta$ is a constant [16]. $N=1000$ is used in these simulations.

The amplitude $A(k)$ is determined by the type of turbulence. Following Ref. [17], it is given by

$$
\begin{aligned}
A^{2}(k)= & B_{r m s}^{2} \frac{(n-1)\left(2 \pi / L_{\max }\right)^{n-1}}{1-\left(L_{\min } / L_{\max }\right)^{n-1}} \delta k \\
& \times \begin{cases}k^{-n} & k \in\left[2 \pi / L_{\max }, 2 \pi / L_{\min }\right], \\
0 & \text { otherwise, }\end{cases}
\end{aligned}
$$

where $n=5 / 3$ corresponds to the Kolmogorov spectrum, $L_{\min }$ and $L_{\max }$ correspond to the minimum and maximum wave lengths, respectively, $\delta k$ is the length of the interval between consecutive values of $k$ defined by the discretization of the $k$ space $\left[\delta k_{i}=\left(10^{\Delta}-1\right) k_{i}\right]$, and $B_{r m s}^{2}=\left\langle\|\mathbf{B}(\mathbf{x})\|^{2}\right\rangle$.

Figure 1 shows the distribution of the $x$ component (top panel) and the squared intensity (bottom panel) of the magnetic field at $\mathbf{x}=\mathbf{0}$. A sample of $2 \times 10^{5}$ realizations of the magnetic field was used for the calculation. 
The parameters used are: $L_{\max }=1 \mathrm{Mpc}, L_{\min }=10^{-3}$ $\mathrm{Mpc}$, and $B_{r m s}=1 \mathrm{nG}$. The coherence length of the field is given by [17],

$$
L_{c}=\frac{(n-1) L_{\max }}{2 n} \times \frac{1-\left(L_{\min } / L_{\max }\right)^{n}}{1-\left(L_{\min } / L_{\max }\right)^{n-1}},
$$

which for this choice of the parameters, in which $L_{\max } \gg$ $L_{\text {min }}$, it takes the value $L_{c} \cong L_{\max } / 5=0.2 \mathrm{Mpc}$. Note that IGMFs similar to this are commonly used in the literature 18 20]. In the plot on the top panel of the figure a Gaussian fit to the distribution is also shown. Although not shown in the figure, the $y$ and $z$ components of the magnetic field also follow a Gaussian distribution. As expected, the mean value corresponding to the distribution on the bottom panel of the figure is $\sim 1 \mathrm{nG}$.
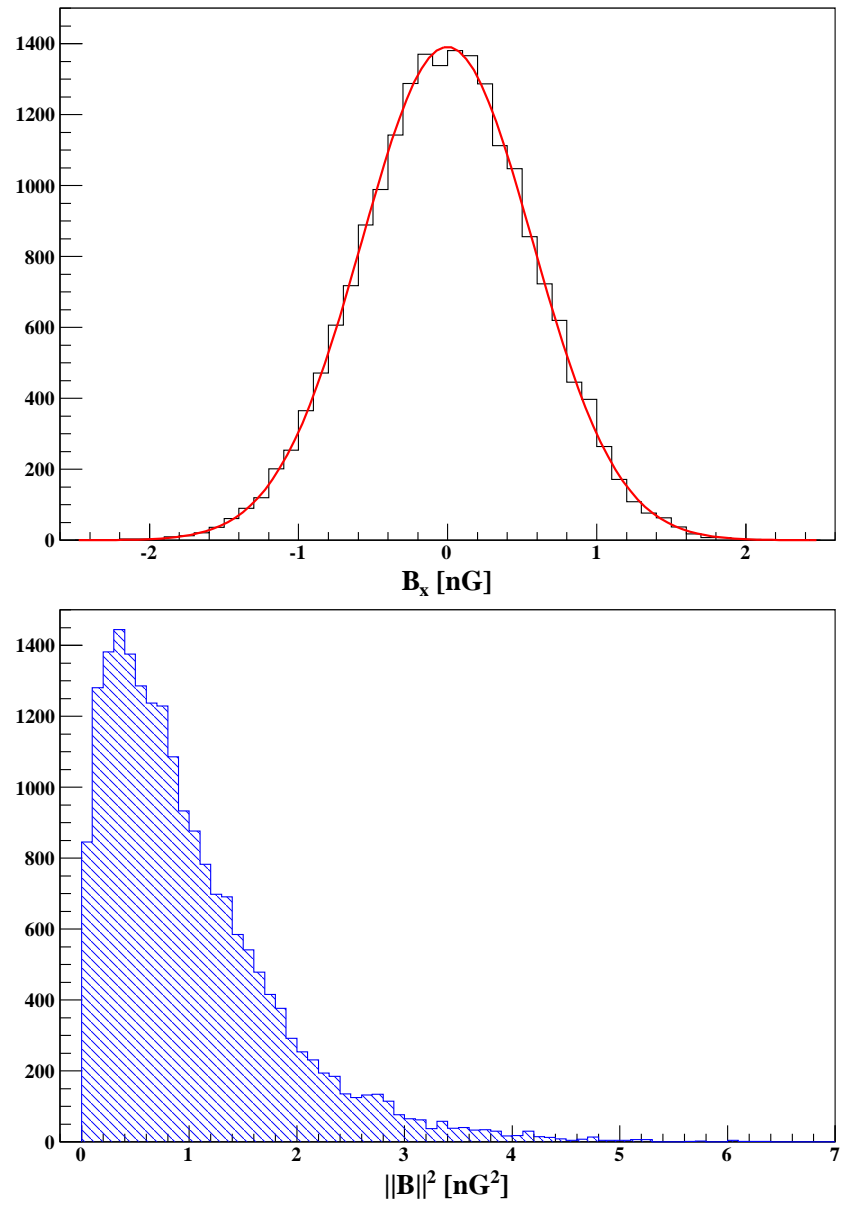

FIG. 1. Top panel: Distribution of the magnetic field $x$ component at $\mathbf{x}=\mathbf{0}$. A Gaussian fit to the distribution is also shown. Bottom panel: Distribution of magnetic field squared intensity at $\mathbf{x}=\mathbf{0}$. Each realization of the magnetic field is such that $B_{r m s}=1 \mathrm{nG}$ and $L_{c} \cong 0.2 \mathrm{Mpc}$.

Figure 2 shows the mean value of the scalar product between the magnetic field evaluated at $\mathbf{x}=\mathbf{0}$ and at $\mathbf{x}=d \cdot \hat{\mathbf{x}}$ ( $\hat{\mathbf{x}}$ is a unit vector pointing to the $x$-axis direction) as a function of the logarithm of the distance to the coordinates origin, $d$. It can be seen from the fig- ure that the average of the scalar product goes to zero for distances of the order of or larger than the coherence length of the magnetic field.

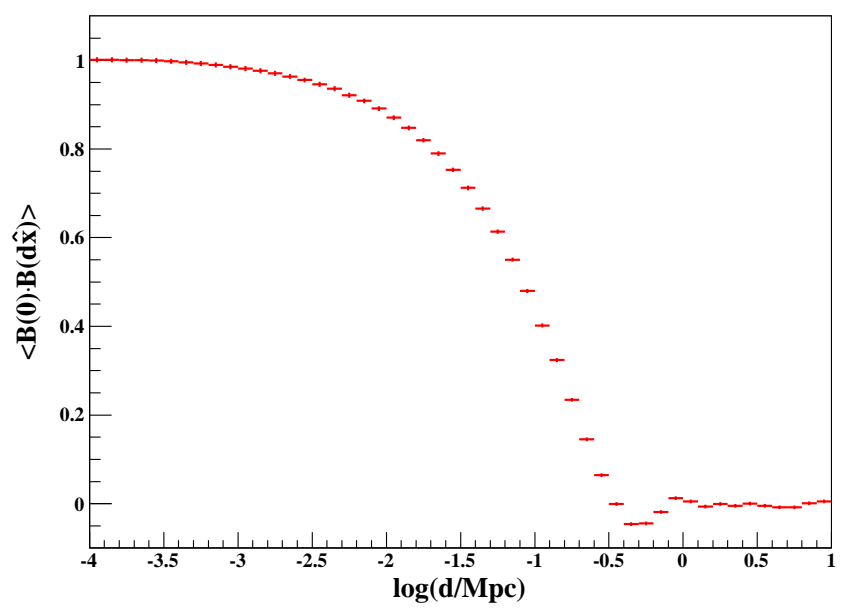

FIG. 2. Mean value of $\mathbf{B}(\mathbf{0}) \cdot \mathbf{B}(d \cdot \hat{\mathbf{x}})$ as a function of the logarithm of the distance to the origin of coordinates $d$. Each realization of the magnetic field is such that $B_{r m s}=1 \mathrm{nG}$ and $L_{c} \cong 0.2 \mathrm{Mpc}$.

As mentioned before, different spatial distributions of cosmic ray sources produce different energy spectra at Earth. Therefore, the energy spectra of a single source as a function of its distance to Earth is required in order to calculate the energy spectrum for a given spatial distribution of sources. For that purpose, CRPropa (version 2.0.4) [21] is used. CRPropa is a program written to propagate nuclei, nucleons, photons, and neutrinos in the intergalactic medium. A complete set of processes undergone by the particles propagating in the intergalactic medium are implemented in that program. The most important processes are photo-pion production (the SOPHIA [22] code, available in CRPropa, is used), pair production, and photodisintegration in both the cosmic microwave background and the extragalactic background light. In particular, it is possible to propagate particles in the presence of an intergalactic magnetic field which can be provided externally. In this calculation, a $10 \mathrm{Mpc}$ side cubic simulation box is used to inject the cosmic ray particles. The simulation box is filled with a turbulent magnetic field calculated by following the method described above. The 3D option is used for the simulation in which the trajectories of charged particles that deviate in the magnetic field are calculated numerically by solving the corresponding differential equations (see Ref. 21] for details). Periodic boundary conditions are used for the simulations. The particles are uniformly distributed in the whole volume and propagated thereafter. They are followed for a time of $4 \mathrm{Gpc} / c$, where $c$ is the speed of light. The particles are recorded every time they enter to a sphere of $1 \mathrm{Mpc}$ around the observer (see Ref. 21] for details on the detection algorithm).

The propagation of protons and iron nuclei is simulated 
for three cases: a null magnetic field, $B_{r m s}=5 \mathrm{nG}$, and $B_{r m s}=10 \mathrm{nG}$. The differential injection spectra for both types of nuclei considered follows a power law with spectral index $\gamma=1\left(\phi \propto E^{-\gamma}\right)$. The energy range extends from $10^{19}$ to $10^{22} \mathrm{eV}$. Note that it is possible to obtain a different injection spectrum by appropriately weighting each particle that reaches the observer. For instance, a power law with spectral index $\gamma=-2$ combined with an exponential cutoff is obtained by weighting each particle with $w_{i}=E_{0, i}^{-1} \times \exp \left(-E_{0, i} / E_{c u t}\right)$, where $E_{0, i}$ is the energy of the parent particle injected at the source corresponding to $i$ th particle observed at Earth and $E_{c u t}$ is the cutoff energy. The output of these simulations is used to calculate the energy spectrum as a function of source distance.

Note that the 3D propagation in a non-null intergalactic magnetic field implemented in CRPropa does not include the effects of the expansion of the Universe and the evolution of the photon backgrounds with redshift. However, these effects become important at energies smaller than a few $10^{19} \mathrm{eV}[14]$. At higher energies they are negligible, because the flux at Earth is dominated by local sources, which is due to the interactions undergone by the cosmic rays during propagation.

\section{ENSEMBLE FLUCTUATIONS AND THE INTERGALACTIC MAGNETIC FIELD}

The injection spectrum considered is given by a power law with an exponential cutoff,

$$
\phi(E)=C_{0} E^{-\gamma} \exp \left(-E / E_{c u t}\right),
$$

where $C_{0}$ is a constant, $\gamma$ is the spectral index, and $E_{c u t}$ is the cutoff energy. From the simulations described in Sec. II it is possible to obtain the number of particles of type $A$ that reach the observer per unit of energy $E$. It is denoted as $K\left(E, A \mid r, E_{c u t}, \gamma, A_{0}, \mathbf{B}\right)$, where $r$ is the injection distance, $A_{0}$ is the type of particles injected by the sources (proton or iron in this work), and $\mathbf{B}$ is the realization of the IGMF considered.

For a given configuration of the IGMF, the flux observed at Earth depends on the density and spatial distribution of the sources. Therefore, the flux corresponding to a given distribution of sources and density can be calculated as

$$
J_{A, m}(E)=C \sum_{i=1}^{N_{s}} \frac{K\left(E, A \mid r_{m, i}, E_{c u t}, \gamma, A_{0}, \mathbf{B}\right)}{r_{m, i}^{2}}
$$

where $C$ is a normalization constant, $N_{s}$ is the number of sources in the volume under consideration, and $r_{m, i}$ is the distance of the $i$ th source of the sample $m(m=1, \ldots, M$, where $M$ is the number of samples considered). Here a constant density of sources is considered. Therefore, $N_{s}$ is sampled from a Poisson distribution with mean value

$$
\mu=\frac{4 \pi}{3} n_{s}\left(R^{3}-r_{m i n}^{3}\right) .
$$

The distance of a given source $r$ is sampled form

$$
P(r)=\frac{3}{R^{3}-r_{\text {min }}^{3}} \times \begin{cases}r^{2} & r \in\left[r_{\text {min }}, R\right] \\ 0 & \text { otherwise }\end{cases}
$$

Note that $r_{\min }$ is the minimum distance allowed for a source [14] and $R$ is taken as $4000 \mathrm{Mpc}$.

The density of UHECR sources is unknown. A lower limit on the local density of sources has been obtained from the analysis of the data taken by The Pierre Auger Observatory 23]. Assuming that the sources are distributed uniformly and by using the two-point autocorrelation function, a lower limit of $\sim(0.06-5) \times 10^{-4}$ $\mathrm{Mpc}^{-3}$ at $95 \%$ confidence level (C.L.) was obtained. If the sources are distributed by following the local matter distribution, the lower limit is $\sim(0.2-7) \times 10^{-4} \mathrm{Mpc}^{-3}$ at $95 \%$ C.L. Therefore, motivated by these results the values of the density of sources $n_{s}=10^{-5} \mathrm{Mpc}^{-3}$ and $n_{s}=10^{-4} \mathrm{Mpc}^{-3}$ are considered in this work.

Figure 3 shows the cosmic ray energy spectra corresponding to different realizations of the distribution of sources. It is assumed in this case that the sources inject only protons into the intergalactic medium. Two different configurations of the IGMF, $B_{r m s}=0$ (top panel) and $B_{r m s}=10 \mathrm{nG}$ (bottom panel), are shown. The density of sources used for the calculation is $n_{s}=10^{-5}$ $\mathrm{Mpc}^{-3}$ and $r_{\min }=3 \mathrm{Mpc}$. Following Ref. [14], we take $E_{\text {cut }}=10^{21} \mathrm{eV}$ and $\gamma=2.2$. From the figure, it can be seen that in both cases the spectra present a strong suppression at $E \sim 10^{19.6} \mathrm{eV}$, which is of the order of the energy threshold of photopion production of protons interacting with low energy photons of the cosmic microwave background. It can also be seen that the ensemble fluctuations of the spectrum increase with energy becoming quite large beyond the beginning of the suppression. This is because, for energies larger than the photopion production threshold, only nearby sources can contribute to the total flux. In this case, there is no evident influence of a non-null IGMF on the energy spectrum.

From a sample of energy spectra, it is possible to estimate the mean value $E[J](E)$ and the relative standard deviation

$$
\varepsilon[J](E)=\frac{\sigma[J](E)}{E[J](E)},
$$

where $\sigma[J](E)$ is the standard deviation of the spectrum.

Figure 4 shows the mean value of the energy spectra (top panel) and the relative standard deviation (bottom panel) as a function of the logarithm of energy. Three cases are considered: $B_{r m s}=0,5$, and $10 \mathrm{nG}$. From the figure, it can be seen that the spectra and the relative standard deviations are practically not affected by the IGMF. Just a small increase on the relative standard deviation corresponding to $B_{r m s}=10 \mathrm{nG}$ can be observed in the energy range from $10^{19}$ to $10^{19.4} \mathrm{eV}$. A comparison between the results obtained in this work and in Refs. 14, 24], for the case of a null IGMF and for sources injecting only protons, is given in Appendix A 

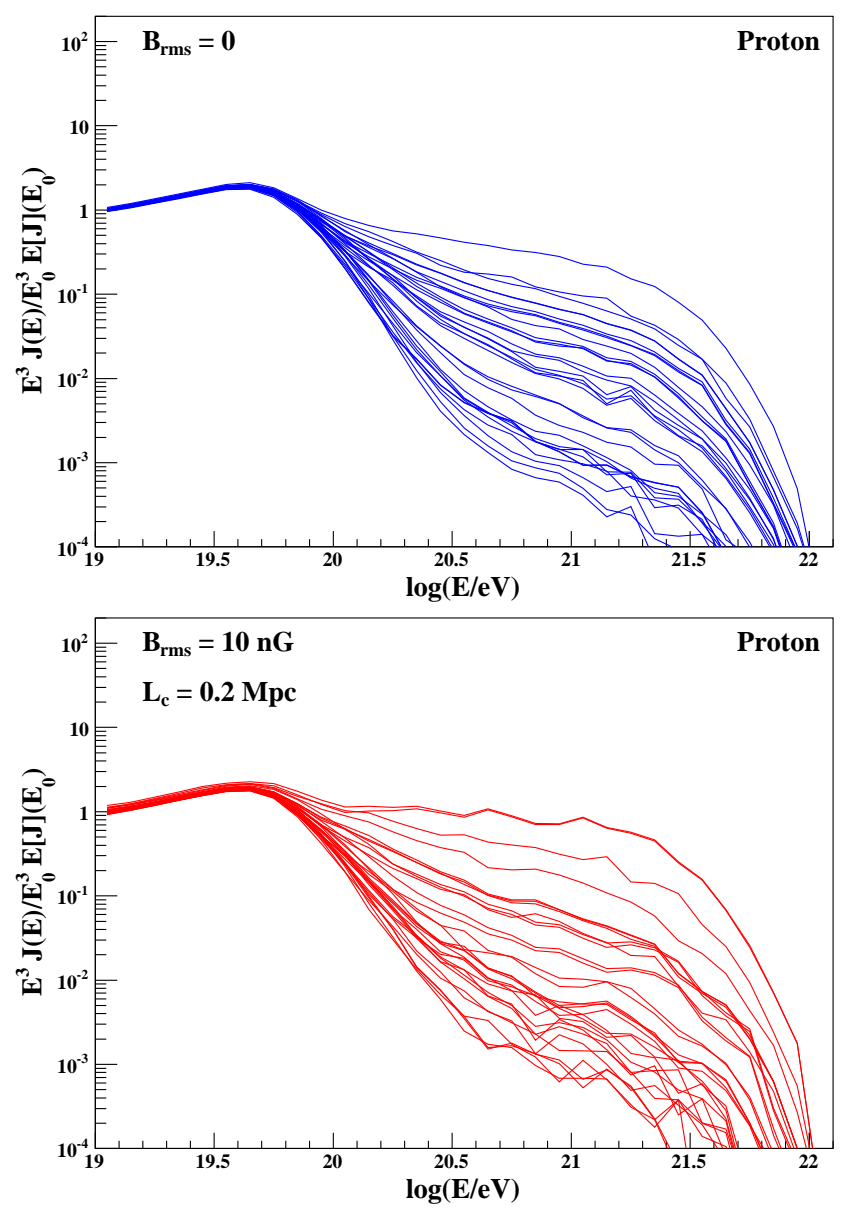

FIG. 3. Cosmic ray energy spectra multiplied by $E^{3}$ as a function of the logarithm of energy for $B_{r m s}=0$ (top panel) and $B_{r m s}=10 \mathrm{nG}$ (bottom panel). The sources inject only protons into the intergalactic medium. The spectra are normalized to their mean value multiplied by $E^{3}$ evaluated at $E_{0}=10^{19} \mathrm{eV}$. The density of sources is $n_{s}=10^{-5} \mathrm{Mpc}^{-3}$, $E_{\text {cut }}=10^{21} \mathrm{eV}, \gamma=2.2$, and $r_{\text {min }}=3 \mathrm{Mpc}$.

Figure 5 shows the cosmic ray energy spectra corresponding to different realizations of the distribution of sources, but in this case the sources inject only iron nuclei into the intergalactic medium. Two different configuration of the intergalactic magnetic field are shown: $B_{r m s}=0$ (top panel) and $B_{r m s}=10 \mathrm{nG}$ (bottom panel). The density of sources used for the calculation is $n_{s}=10^{-5} \mathrm{Mpc}^{-3}$ and $r_{\text {min }}=3 \mathrm{Mpc}$. In this case we take $E_{\text {cut }}=10^{21} \mathrm{eV}$ and $\gamma=2[14]$. Note that it is evident that for the $B_{r m s}=10 \mathrm{nG}$ case the ensemble fluctuations are much larger than for $B_{r m s}=0$.

Figure 6] shows the mean value of the energy spectra (top panel) and the relative standard deviation (bottom panel) as a function of the logarithm of energy for the case in which the sources inject only iron nuclei into the intergalactic medium. In this case the presence of a non-null IGMF modifies the shape of the mean energy spectrum. Also the ensemble fluctuations increase significantly at low energy. From the figure, it can be
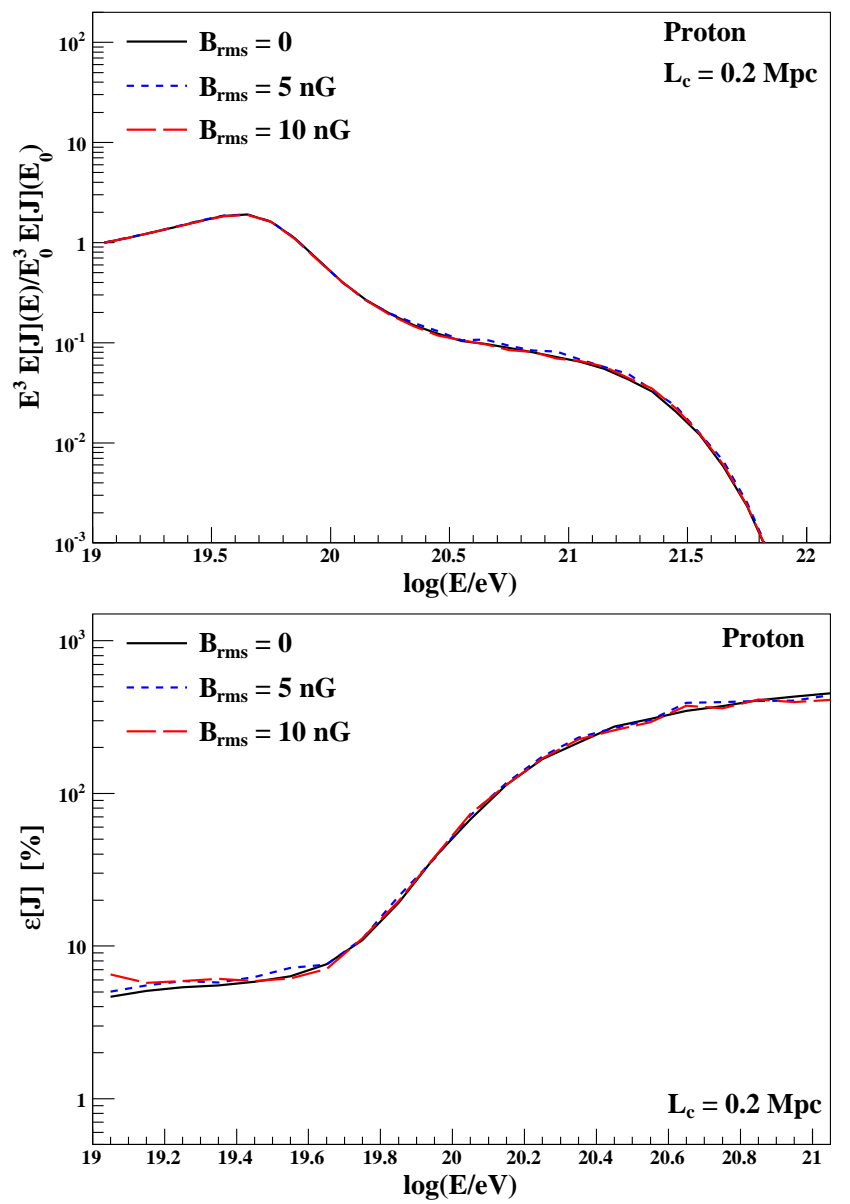

FIG. 4. Top panel: Mean value of the energy spectrum multiplied by $E^{3}$ as a function of the logarithm of energy. Bottom panel: Relative standard deviation as a function of the logarithm of energy. The sources inject only protons and the IGMF is such that $B_{r m s}=0,5$, and $10 \mathrm{nG}$. The density of sources is $n_{s}=10^{-5} \mathrm{Mpc}^{-3}, E_{\text {cut }}=10^{21} \mathrm{eV}, \gamma=2.2$, and $r_{\min }=3 \mathrm{Mpc}$.

seen that at $E \sim 10^{19} \mathrm{eV}$ the relative standard deviation for $B_{r m s} \geq 5 \mathrm{nG}$ is more than one order of magnitude larger than for $B_{r m s}=0$. The relative standard deviation curves for non-null IGMF get closer to the curve corresponding to a null IGMF when energy increases. That is in such a way that for $E \gtrsim 10^{20} \mathrm{eV}$ the differences are small. A comparison between the results obtained in this work and in Refs. [14, 24], for the case of a null IGMF and for sources injecting only iron nuclei, is given in Appendix A

The increase of the ensemble fluctuations of the spectrum at low energies can be understood from the fact that, in the presence of a non-null IGMF, cosmic rays travel larger distances before reaching the observer due to the deviations suffered by the interaction with the magnetic field. Therefore, the sources that can contribute to the flux observed at Earth are closer than in the case of a null IGMF. In fact, at any given energy, fewer sources contribute to the spectrum, increasing its 

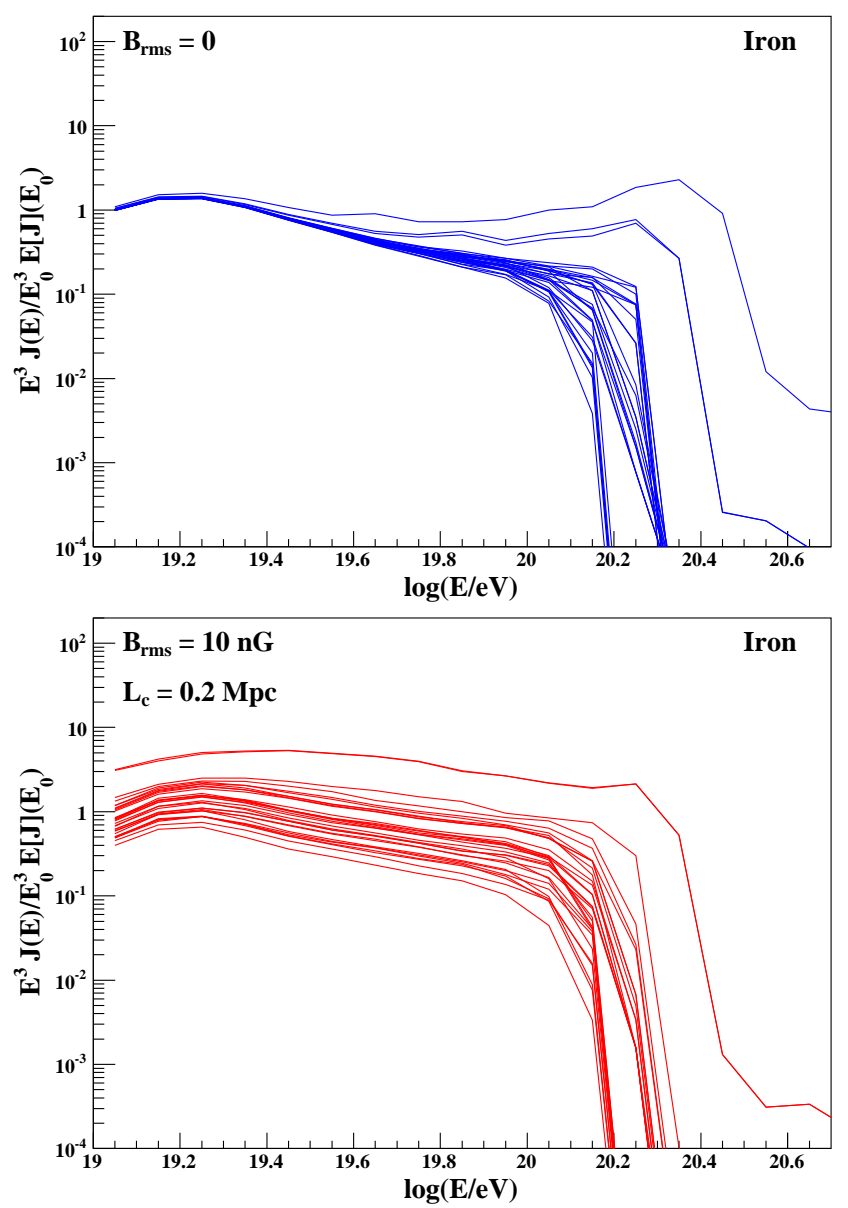

FIG. 5. Cosmic ray energy spectra multiplied by $E^{3}$ as a function of the logarithm of energy for $B_{r m s}=0$ (top panel) and $B_{r m s}=10 \mathrm{nG}$ (bottom panel). The sources inject only iron nuclei into the intergalactic medium. The spectra are normalized to the mean value multiplied by $E^{3}$ evaluated at $E_{0}=10^{19} \mathrm{eV}$. The density of sources is $n_{s}=10^{-5} \mathrm{Mpc}^{-3}$, $E_{\text {cut }}=10^{21} \mathrm{eV}, \gamma=2$, and $r_{\min }=3 \mathrm{Mpc}$.

ensemble fluctuations, and this effect is more notorious as the energy decreases. Figure 7 shows the distribution of source distance $D$ corresponding to cosmic rays that reach the observer for sources that inject only iron nuclei into the intergalactic medium. In the top panel of the figure, the distributions corresponding to a null IGMF and $B_{r m s}=10 \mathrm{nG}$, for $E \in\left[10^{19}, 10^{19.5}\right] \mathrm{eV}$, are shown. It can be seen that the distribution for $B_{r m s}=10 \mathrm{nG}$ is strongly peaked at $\sim 40 \mathrm{Mpc}$ and then goes to zero quite fast. Meanwhile, for the case of a null IGMF, the distribution is quite flat, allowing distant sources to contribute to the spectrum. The bottom panel of the figure shows the distributions of source distance but for $E \geq 10^{20}$ $\mathrm{eV}$. In this case, both distributions have a maximum at $D=0$ and go to zero quite fast. However, the one corresponding to $B_{r m s}=10 \mathrm{nG}$ is more concentrated around zero, which means that also in this case the sources that dominate the spectrum for $B_{r m s}=10 \mathrm{nG}$ are closer than for the case of a null IGMF. Note that in this case the
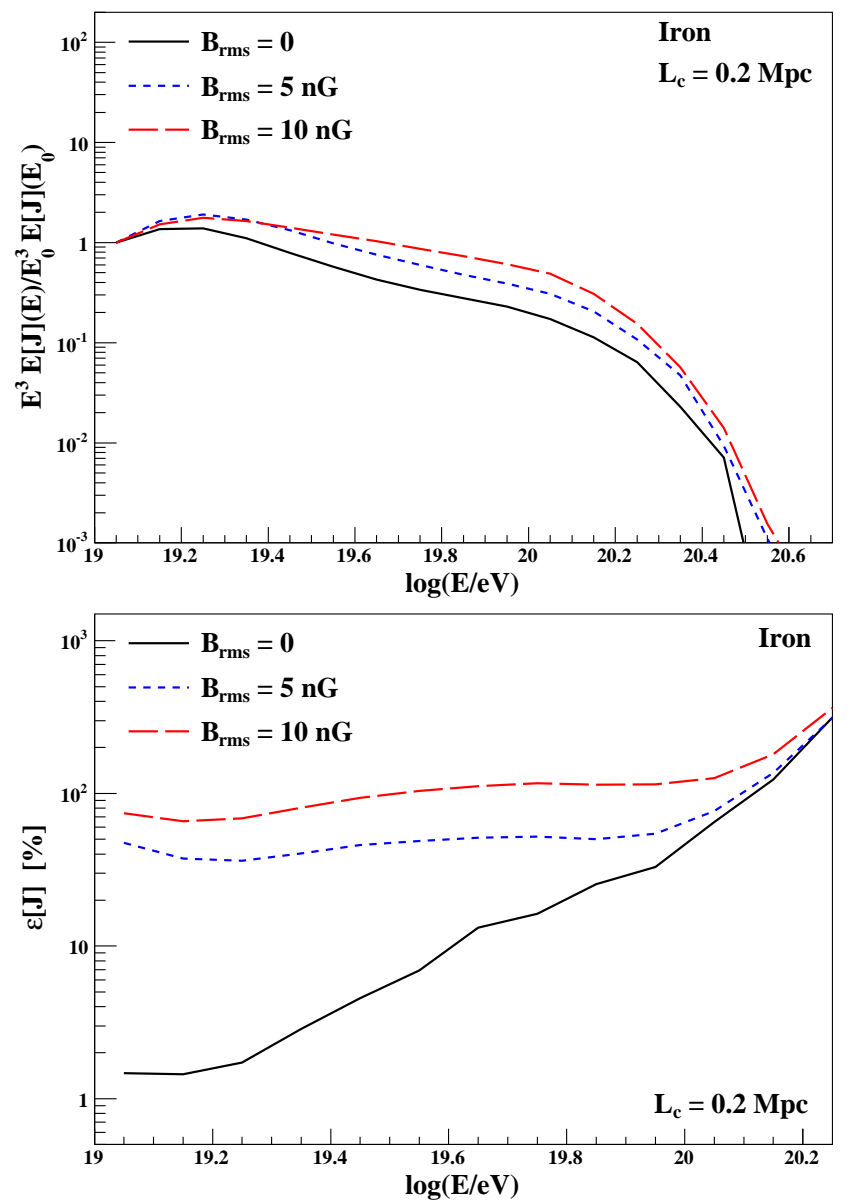

FIG. 6. Top panel: Mean value of the energy spectrum multiplied by $E^{3}$ as a function of the logarithm of energy. Bottom panel: Relative standard deviation as a function of the logarithm of energy. The sources inject only iron nuclei and the intergalactic magnetic field is such that $B_{r m s}=0,5$, and 10 $\mathrm{nG}$. The density of sources is $n_{s}=10^{-5} \mathrm{Mpc}^{-3}, E_{\text {cut }}=10^{21}$ $\mathrm{eV}, \gamma=2$, and $r_{\text {min }}=3 \mathrm{Mpc}$.

difference is smaller compared to the case corresponding to lower energies. This is due to the fact that charged particles of larger energies are less affected by the IGMF. For protons, the effect is negligible.

For the case in which the sources inject heavy nuclei, also the composition profile at Earth is affected by the IGMF. The composition observed at Earth can be characterized by the average of the logarithm of the mass number $A$, which is given by

$$
\langle\ln A\rangle(E)=\frac{\sum_{A} \ln A J_{A}(E)}{\sum_{A} J_{A}(E)} .
$$

Note that $\langle\ln A\rangle$ also depends on the realization of the spatial distribution of sources.

As mentioned before, the cosmic rays travel larger distances in the presence of a non-null IGMF. Therefore, the probability to suffer a given interaction with the radiation field of the intergalactic medium is higher. In 

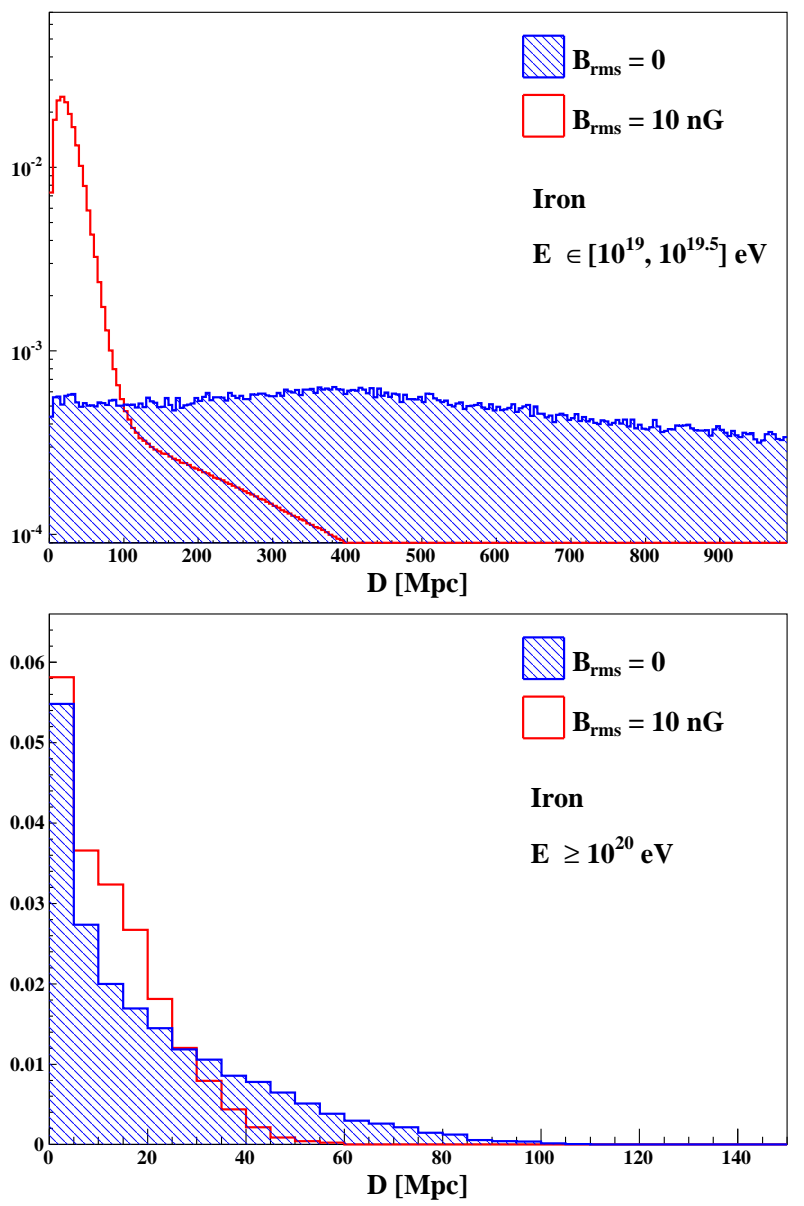

FIG. 7. Distribution of source distance for a null IGMF and for $B_{r m s}=10 \mathrm{nG}$. The sources inject only iron nuclei. The spectral index is $\gamma=2$ and $E_{c u t}=10^{21} \mathrm{eV}$. Top panel: Low energy interval $\left(E \in\left[10^{19}, 10^{19.5}\right] \mathrm{eV}\right)$. Bottom panel: High energy interval $\left(E \geq 10^{20} \mathrm{eV}\right)$.

particular, they can suffer photodisintegration. As a result, a lighter composition is expected at Earth [25]. The top panel of Fig. 8 shows the mean value of $\langle\ln A\rangle$ as a function of the logarithm of energy. From this plot, it can be seen that the composition gets lighter for increasing values of $B_{r m s}$.

The ensemble fluctuations of the composition profile are also affected by the IGMF. The bottom panel of Fig. 8 shows the relative standard deviation of $\langle\ln A\rangle$ as a function of the logarithm of energy. As in the case of the energy spectrum, the ensemble fluctuations are larger for increasing values of $B_{r m s}$.

Note that also, in the case of the composition profile and its relative standard deviation, the curves corresponding to non-null IGMFs get closer to the one corresponding to a null IGMF for increasing values of energy. As mentioned before, this is caused by the diminution of the magnetic field effects suffered by the charged cosmic rays for increasing values of energy.

In Ref. [14], it is shown that the relative standard de-
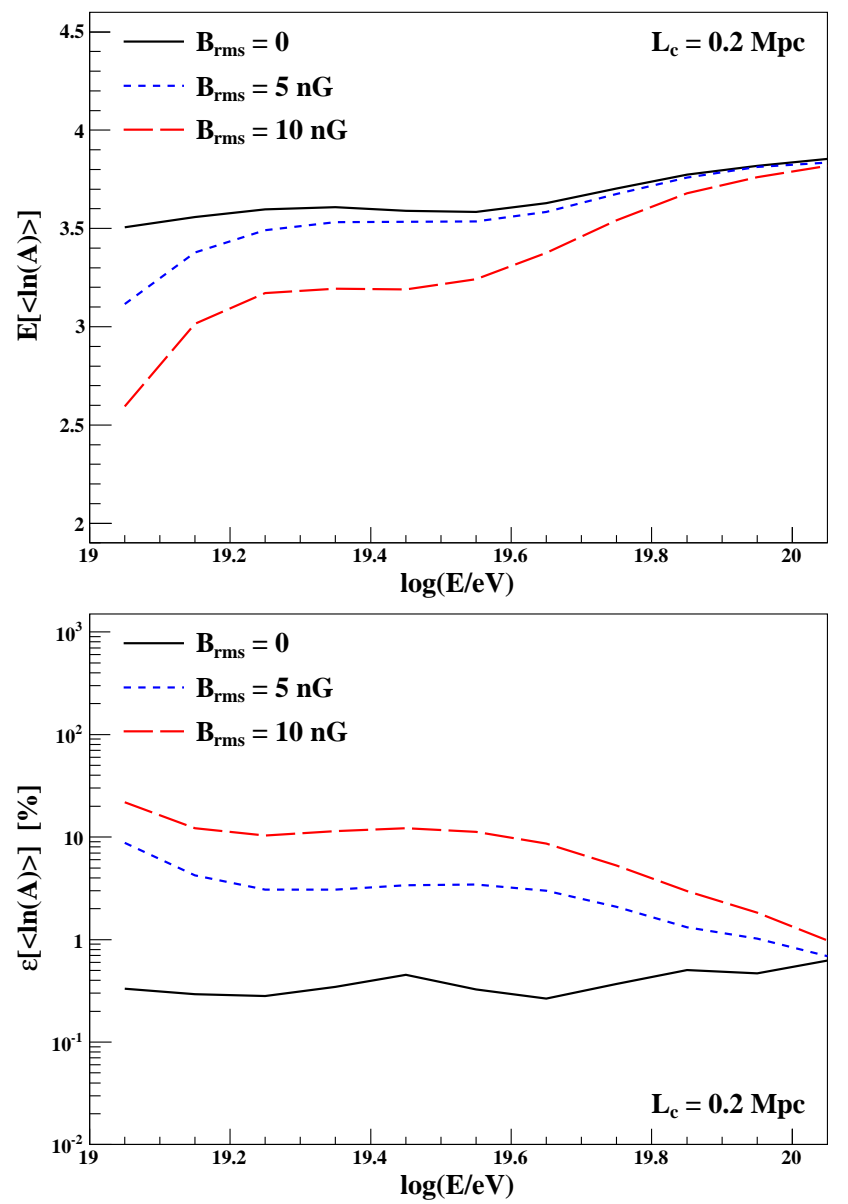

FIG. 8. Top panel: Mean value of $\langle\ln A\rangle$ as a function of the logarithm of energy. Bottom panel: Relative standard deviation of $\langle\ln A\rangle$ as a function of the logarithm of energy. The spectral index is $\gamma=2, E_{\text {cut }}=10^{21} \mathrm{eV}, r_{\text {min }}=3 \mathrm{Mpc}$, and $n_{s}=10^{-5} \mathrm{Mpc}^{-3}$.

viation for a null IGMF is smaller for larger values of $r_{\text {min }}$. Figure 9 shows the relative standard deviation for $r_{\text {min }}=10 \mathrm{Mpc}$, density of sources $n=10^{-5} \mathrm{Mpc}^{-3}$, and for all configurations of the IGMF considered before. The top panel shows the results for protons and the bottom panel the ones for iron nuclei. The relative standard deviations are smaller compared with the $r_{\text {min }}=3 \mathrm{Mpc}$ case, for all configurations of the IGMF considered. The reason for that is that, as mentioned before, sources close to the observer introduce large ensemble fluctuations because the distribution of distances is $\propto r^{2}$. Therefore, when sources with $r \in[3,10] \mathrm{Mpc}$ are removed, the ensemble fluctuations decrease.

The ensemble fluctuations of both energy spectrum and composition profile depend on the density of sources. The larger the density of sources, the smaller the ensemble fluctuations. Figure 10] shows the relative standard deviation for sources that inject only protons (top panel) or iron nuclei (bottom panel) for $n_{s}=10^{-4} \mathrm{Mpc}^{-3}$ and $r_{\text {min }}=3 \mathrm{Mpc}$ for both cases. By comparing with the bot- 

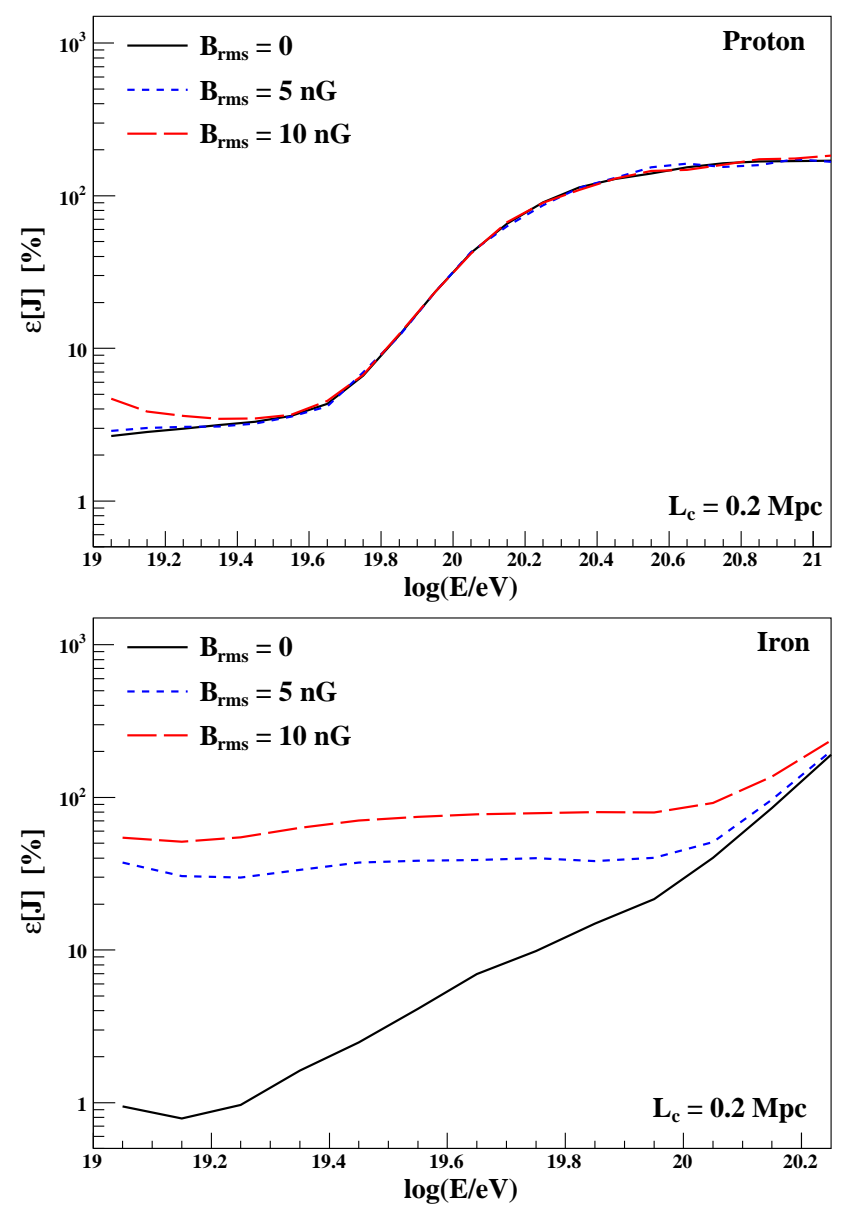

FIG. 9. Relative standard deviation as a function of the logarithm of energy. The sources inject only protons (top panel) or iron nuclei (bottom panel). The IGMF is such that $B_{r m s}=0$, 5 , and $10 \mathrm{nG}$. The density of sources is $n_{s}=10^{-5} \mathrm{Mpc}^{-3}$ and $r_{\text {min }}=10 \mathrm{Mpc}$. The spectral index is $\gamma=2.2$ for iron nuclei and $\gamma=2$ for protons, and $E_{c u t}=10^{21} \mathrm{eV}$ for both cases.

tom panels of Figs. 4 and 6, it is clear that the relative standard deviations decreases in all cases. The relative standard deviation for $n_{s}=10^{-5} \mathrm{Mpc}^{-3}$ is $\sim 30-35$ times larger than the ones corresponding to $n_{s}=10^{-4}$ $\mathrm{Mpc}^{-3}$, for all cases considered. Note that for the cases corresponding to $r_{\min }=10 \mathrm{Mpc}$ this ratio is in the reported range but slightly smaller than the one corresponding to the same cases but for $r_{\min }=3 \mathrm{Mpc}$.

\section{INFLUENCE ON THE INTERPRETATION OF THE EXPERIMENTAL DATA}

It is a common practice to fit the cosmic ray energy spectrum, obtained experimentally, with the mean value of the flux corresponding to a given astrophysical model. In order to study the effects of the IGMF on this type of practice, let us normalize the mean value of the flux, evaluated at the lowest value of energy considered $\left(E_{0}=10^{19.05} \mathrm{eV}\right)$, to the one corresponding to a given re-
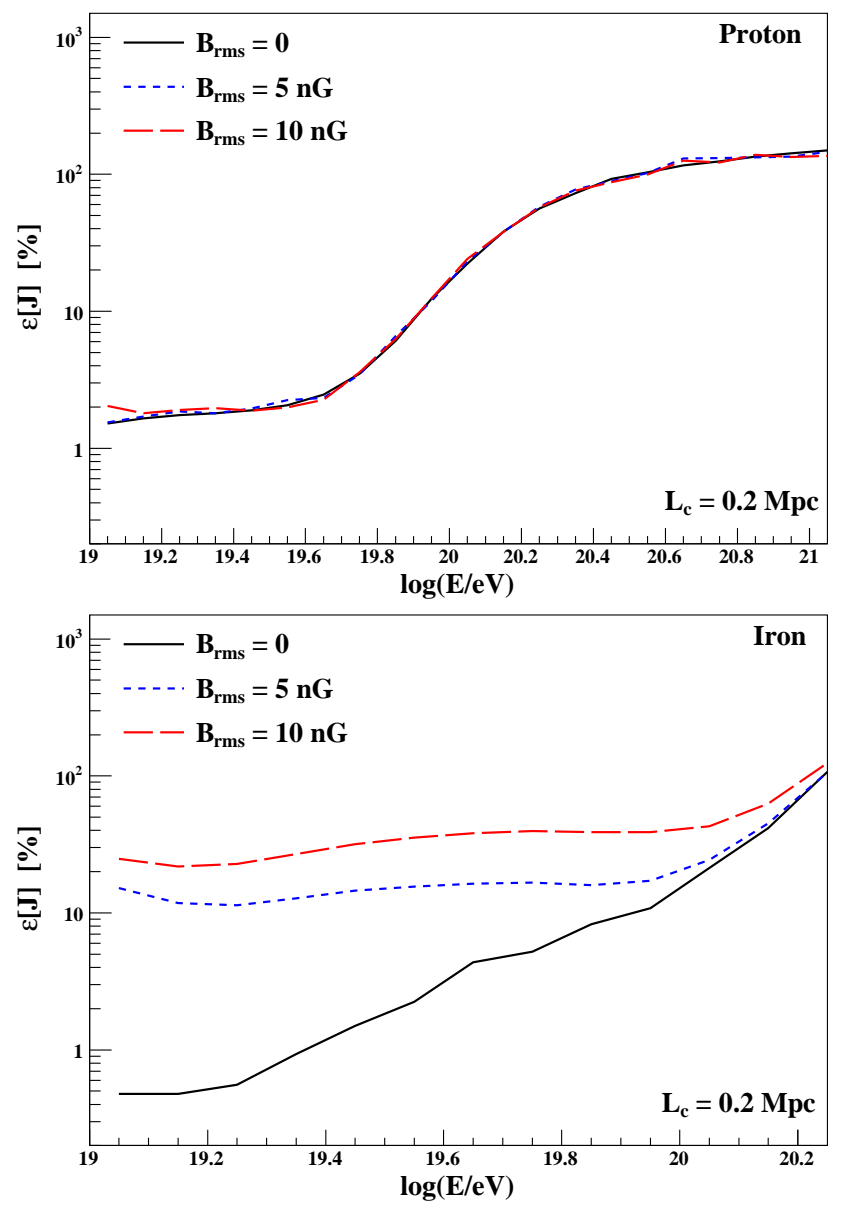

FIG. 10. Relative standard deviation as a function of the logarithm of energy. The sources inject only protons (top panel) or iron nuclei (bottom panel). The IGMF is such that $B_{r m s}=0,5$, and $10 \mathrm{nG}$. The density of sources is $n_{s}=10^{-4}$ $\mathrm{Mpc}^{-3}$ and $r_{\min }=3 \mathrm{Mpc}$. The spectral index is $\gamma=2.2$ for iron nuclei and $\gamma=2$ for protons, and $E_{\text {cut }}=10^{21} \mathrm{eV}$ for both cases.

alization of the source distribution evaluated at the same energy. The flux obtained in this way is given by

$$
\tilde{J}(E)=\frac{J\left(E_{0}\right)}{E[J]\left(E_{0}\right)} E[J](E),
$$

which is taken as an approximated representation of the real spectrum. Note that this procedure corresponds to change the luminosity of the sources; i.e., if $J\left(E_{0}\right)>$ $E[J]\left(E_{0}\right)$, a larger luminosity, compared with the true one, is inferred. The top panel of Fig. 11 shows the distributions corresponding to the ratio between the inferred luminosity and the true one for sources injecting iron nuclei with the spectral index and cutoff energy considered before, $r_{\text {min }}=3 \mathrm{Mpc}$, and $n_{s}=10^{-5} \mathrm{Mpc}^{-3}$. The IGMF is such that $B_{r m s}=0$ and $B_{r m s}=10 \mathrm{nG}$. From the figure, it is easy to see that for the non-null IGMF case the inferred luminosity can be $\sim 4$ times larger or smaller than the true one. In contrast, for the case of a null IGMF the inferred luminosity differs from the true 
one in less than $\sim 15 \%$. The increase of the error on the inferred luminosity for a non null IGMF is due to the larger ensemble fluctuations compared with the ones corresponding to a null IGMF.
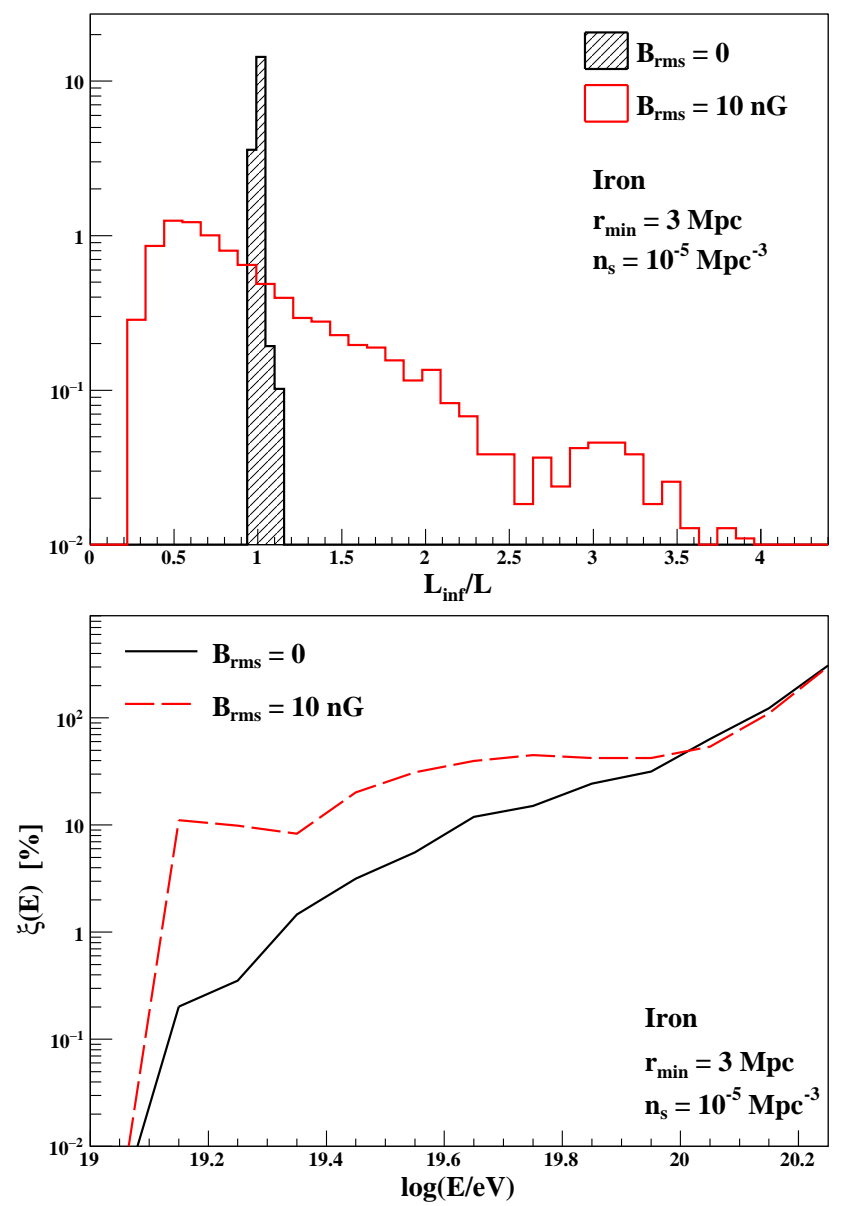

FIG. 11. Top panel: Distributions of the ratio between the inferred luminosity and the true one for sources injecting iron nuclei in the presence of a null and a non-null IGMF. The distributions are normalized to one. Bottom panel: $\xi$ as a function of the logarithm of energy. In this case, $n_{s}=10^{-5}$ $\mathrm{Mpc}^{-3}$ and $r_{\min }=3 \mathrm{Mpc}$.

In order to estimate the relative error corresponding to the use of the mean value of the flux to represent the energy spectrum, obtained for a particular realization of the position of the sources, the following quantity is introduced

$$
\xi(E)=\frac{\sigma[\Delta J](E)}{E[J](E)},
$$

where $\Delta J(E)=J(E)-\tilde{J}(E)$. Note that, by definition, $\xi\left(E_{0}\right)=0$. The bottom panel of Fig. 11] shows $\xi$ as a function of the logarithm of the energy. It can be seen that, for energies smaller than $10^{20} \mathrm{eV}$, the relative error corresponding to a non-null IGMF case is larger than the one corresponding to the null IGMF case. The difference is larger for energies of the order of $10^{19.2} \mathrm{eV}$. For energies larger than $10^{20} \mathrm{eV}$, the differences are small.
Figure 12 shows the results obtained by increasing the density of sources to $n_{s}=10^{-4} \mathrm{Mpc}^{-3}$. From the top panel of the figure, it can be seen that, in this case, the inferred luminosity can be $\sim 2$ times larger or smaller than the true one. For the case of a null IGMF the inferred luminosity differs from the true one by less than $\sim 3 \%$. As expected, the error on the determination of the luminosity is reduced, but it is still quite large for the case of a non-null IGMF. From the bottom panel of Fig. 12,
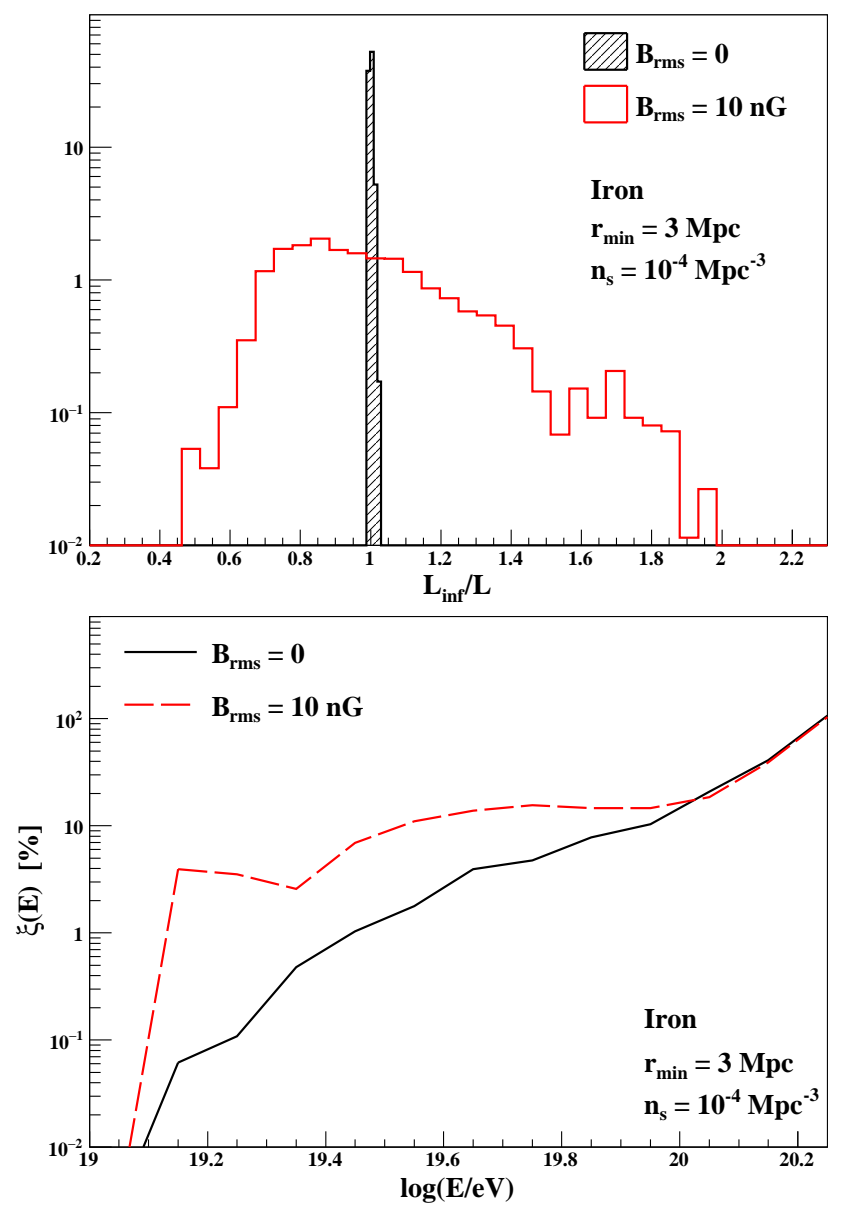

FIG. 12. Top panel: Distributions of the ratio between the inferred luminosity and the true one for sources injecting iron nuclei in the presence of a null and a non-null IGMF. The distributions are normalized to one. Bottom panel: $\xi$ as a function of the logarithm of energy. In this case, $n_{s}=10^{-4}$ $\mathrm{Mpc}^{-3}$ and $r_{\min }=3 \mathrm{Mpc}$.

it can be seen that $\xi$ is smaller than in the previous case for both scenarios considered. However, also in this case the difference between $\xi$ for a null and a non-null IGMF is still quite large in the region of $10^{19.2} \mathrm{eV}$. As in the previous case, the difference is small for energies larger than $10^{20} \mathrm{eV}$. 


\section{PROSPECT OF OBSERVATION}

The study of the ensemble fluctuations requires a large statistics at the highest cosmic ray energies. The Extreme Universe Space Observatory on board the Japanese Experimental Module (JEM-EUSO) is ideal for this type of study as shown in Ref. [24]. JEM-EUSO will consist of the installation of a fluorescence telescope on the International Space Station pointing towards Earth's surface [26]. The atmospheric air showers originated by the interaction of the cosmic rays with the air molecules generate fluorescence and Cherenkov light. A fraction of this light can be collected by the telescope, allowing for the reconstruction of the main shower parameters (arrival direction, primary energy, etc.). The telescope can be operated in nadir mode (pointing in the nadir direction) or tilted mode (forming an angle with the nadir direction). The exposure in tilted mode increases quite fast with the nadir angle, but the energy threshold also increases. Note that the annual exposure of JEM-EUSO in one year of data taking in Nadir mode is $9-10$ times larger than that of The Pierre Auger Observatory [27]. This will allow the collection of a cosmic ray sample of unprecedented statistics at the highest energies.

It is worth mentioning that the integral cosmic ray energy spectrum, defined as

$$
I(E)=\int_{E}^{\infty} d E^{\prime} J\left(E^{\prime}\right),
$$

also presents ensemble fluctuations. Figure 13 shows the relative standard deviation of the integral energy spectrum compared with the statistical fluctuations of the integral energy spectrum for 5 years of data taking of JEMEUSO in nadir mode. For the latter, Poissonian fluctuations are assumed. Also the energy spectrum obtained by The Pierre Auger Observatory [28] and the JEM-EUSO exposure calculated in Ref. [27] are used. The calculation is done for the two models considered before in which the sources inject only protons or iron nuclei. The shadowed regions represent the range of the relative standard deviation of the integral spectrum for $B_{r m s} \in[0,10] \mathrm{nG}$. The top panels of the figure correspond to $n_{s}=10^{-5} \mathrm{Mpc}^{-3}$, and the bottom panels correspond to $n_{s}=10^{-4} \mathrm{Mpc}^{-3}$. Also, the left panels correspond to $r_{\min }=3 \mathrm{Mpc}$, and the right panels correspond to $r_{\min }=10 \mathrm{Mpc}$.

From the figure, it can be seen that for $n_{s}=10^{-5}$ $\mathrm{Mpc}^{-3}$ the ensemble fluctuations are larger than the Poissonian fluctuations, in the whole energy range, for both models considered. For $n_{s}=10^{-4} \mathrm{Mpc}^{-3}, r_{\min }=$ 3 , and protons, also the ensemble fluctuations are larger than the Poissonian fluctuations, in the whole energy range considered. However, for iron nuclei and small values of the IGMF intensity, the ensemble fluctuations are larger than the Poissonian fluctuations only if $E>10^{19.3}$ $\mathrm{eV}$. For $n_{s}=10^{-4} \mathrm{Mpc}^{-3}, r_{\min }=10$, and protons, the Poissonian fluctuations are of the order of or even larger than the ensemble fluctuations in the whole energy range.
However, for iron nuclei and small values of the IGMF intensity, the ensemble fluctuation are larger than the Poissonian fluctuations for $E>10^{19.5} \mathrm{eV}$. Note that for the iron injection model and $B_{r m s} \gtrsim 5 \mathrm{nG}$ the ensemble fluctuations are larger than the Poissonian fluctuations for all cases considered. Therefore, no null IGMF and heavy primaries at the highest energies favor the observation of the ensemble fluctuations with JEM-EUSO. Also, it is possible that the lifetime of the JEM-EUSO mission may be extended to more than 5 years. It will be important for the observation of the ensemble fluctuations.

\section{CONCLUSIONS}

In this work, the influence of a turbulent IGMF on the ensemble fluctuations of the cosmic ray energy spectrum and composition profile has been studied. Sources injecting only protons and only iron nuclei have been considered. Turbulent IGMFs of coherence length $L_{c} \cong 0.2$ Mpc and intensities $B_{r m s}=5$ and $10 \mathrm{nG}$ have been taken into account. It has been found that the influence of such magnetic fields on the ensemble fluctuations in the proton model is almost negligible. For the case of iron nuclei, it has been found that, at energies of the order of a few $10^{19} \mathrm{eV}$ and for $B_{r m s} \geq 5 \mathrm{nG}$, the ensemble fluctuations are more than one order of magnitude larger compared with the case of a null IGMF. This difference decreases with increasing energies due to the fact that cosmic rays of higher energies are less affected by the same magnetic field configuration. The same behavior has been found for the ensemble fluctuations of the composition profile. Also, the composition becomes lighter for increasing values of the IGMF intensity. As in the case of the ensemble fluctuations of the energy spectrum, the differences with the null IGMF case decrease for increasing values of energy. The increase of the ensemble fluctuations can be understood from the fact that cosmic rays propagating in a non-null IGMF are injected by sources that are closer. This is caused by the increase of the path length of the particles combined with their interactions with the low energy photons of the radiation field present in the intergalactic medium. The lighter composition can be explained by the increase of the path length traveled by the particles in the presence of a non-null IGMF.

It has been found that, for sources injecting heavy primaries, the presence of a non-null IGMF largely increases the uncertainty on the inferred luminosity of the sources, when the mean value of the flux is used to represent the energy spectrum corresponding to a particular realization of the position of the sources. Also, the uncertainty on the flux shape is in some energy regions much larger for the case of a non-null IGMF. These uncertainties become smaller for increasing values of the density of sources. However, for the cosmic ray and IGMF models considered in this work, even for densities of the sources of the order of $n_{s}=10^{-4} \mathrm{Mpc}^{-3}$, these uncertainties are quite large. 

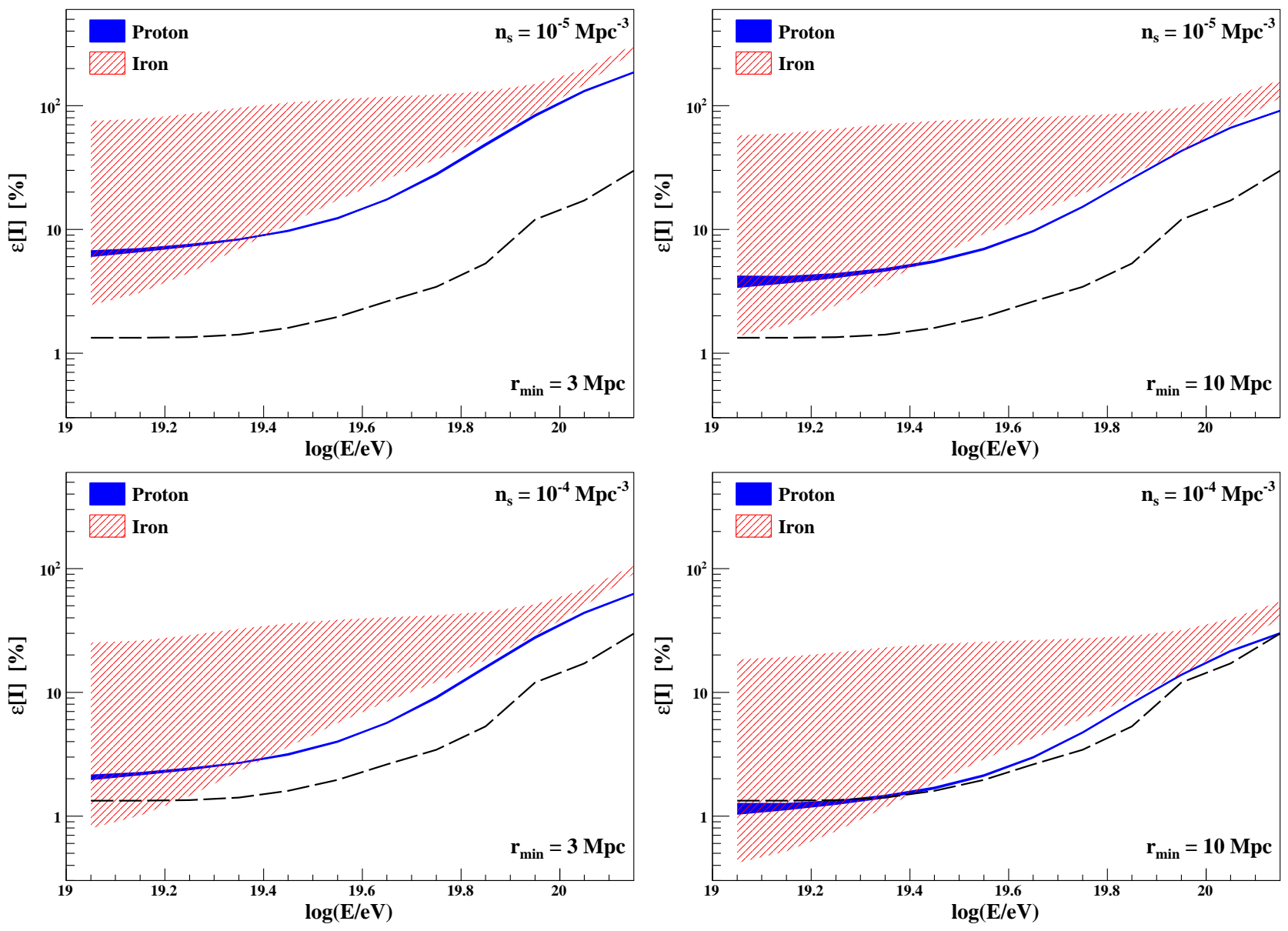

FIG. 13. Relative standard deviation of the integral cosmic ray energy spectrum as a function of the logarithm of energy. Solid dashed line: Statistical fluctuations of the energy spectrum for 5 years of data taking of JEM-EUSO in nadir mode.

Regarding the possible observation with the next generation of cosmic ray observatories like JEM-EUSO, it has been demonstrated that the ensemble fluctuations on the integral energy spectrum will be easier to be observed for models including the injection of heavy nuclei combined with a non-null IGMF. In any case, at least for the models considered in this work, it seems possible to observe ensemble fluctuations, even for density of sources as large as $10^{-4} \mathrm{Mpc}^{-3}$, in 5 years of observation with the JEM-EUSO telescope in nadir mode. Note that the extension of the lifetime of the JEM-EUSO mission will be of great benefit for the observation of the ensemble fluctuations.

\section{ACKNOWLEDGMENTS}

A.D.S. is a member of the Carrera del Investigador Científico of CONICET, Argentina. This work is supported by CONICET PIP 114-201101-00360 and ANPCyT PICT-2011-2223, Argentina. This work was partially supported by PAPIIT-UNAM, Red FAE, and Red
CyTE from CONACyT México.

\section{Appendix A: Comparison with other calculations}

Figure 14]shows the comparisons corresponding to protons (top panel) and iron nuclei (bottom panel) between the relative standard deviation $\varepsilon[J]$ obtained in this work and in Refs. 14, 24]. The calculations correspond to a null IGMF, density of sources $n_{s}=10^{-5} \mathrm{Mpc}^{-3}$, and $r_{\text {min }}=3 \mathrm{Mpc}$. The spectral indexes are $\gamma=2.2$ and $\gamma=2$ for protons and iron nuclei, respectively. The cutoff energy is $E_{\text {cut }}=10^{21} \mathrm{eV}$ in both cases.

From the figure, it can be seen that, although the curves obtained in this work and the ones from Ref. 14, 24] have a similar trend, there are differences. In particular, for protons at low energies, $E \in\left[10^{19}, 10^{19.8}\right] \mathrm{eV}$, both calculations differ in less than $\sim 18 \%$ and for energies larger than $10^{19.8} \mathrm{eV}$ the difference between both calculations increases reaching values of $\sim 70 \%$. For the case of iron nuclei, the differences reach values of at most $\sim 60 \%$ in the energy range considered. As can be seen from the bottom panel of the figure, the larger differences are given at lower energies $\left(E \lesssim 10^{19.4} \mathrm{eV}\right)$. 

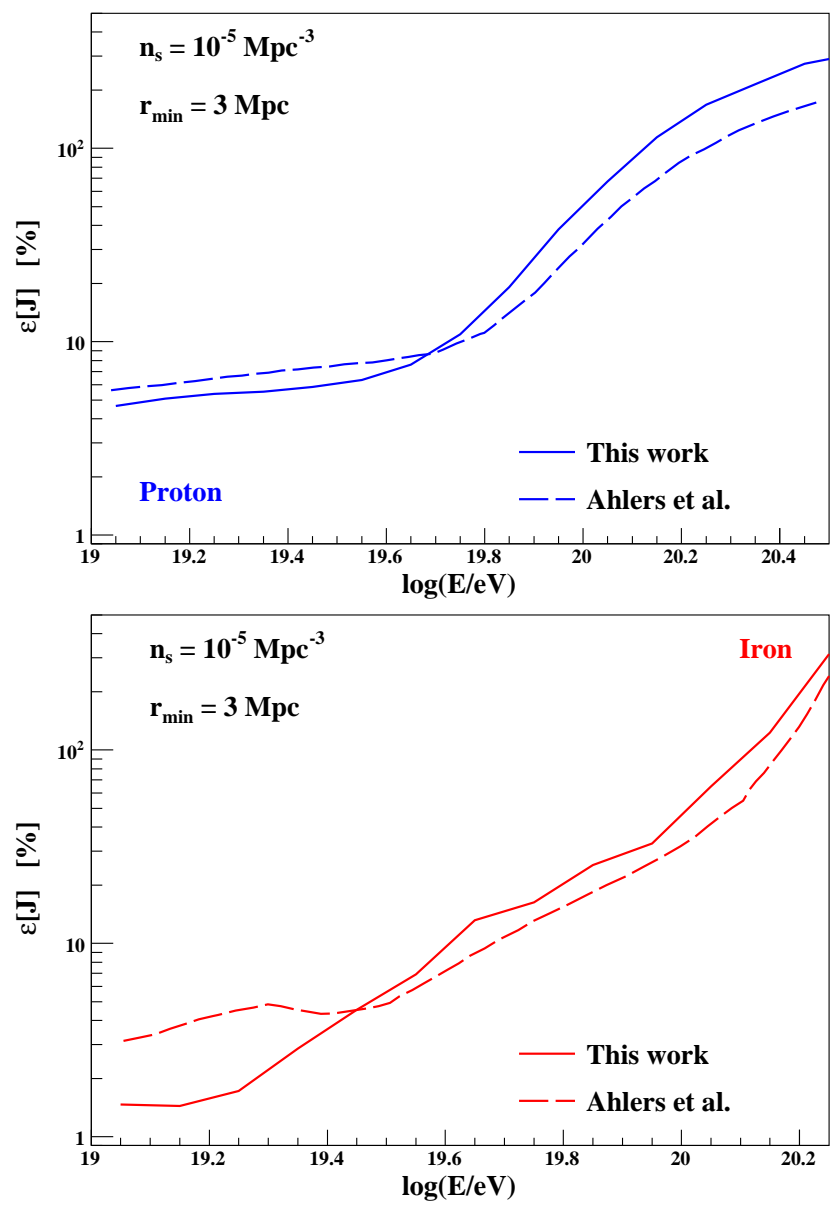

FIG. 14. Relative standard deviation as a function of the logarithm of energy obtained in this work (solid lines) and in Refs. [14, 24] (dashed lines), corresponding to protons (top panel) and iron nuclei (bottom panel).

[1] K. Kotera and A. Olinto, Annu. Rev. Astron. Astrophys. 49, 119 (2011).

[2] A. M. Hillas, arXiv:astroph/0607109.

[3] W. Apel et al., Phys. Rev. Lett. 107, 171104 (2011).

[4] J. Abraham et al., Phys. Rev. Lett. 104, 091101 (2010).

[5] A. Letessier-Selvon for the Pierre Auger Collaboration, Braz. J. Phys. 44, 560 (2014).

[6] R. Abbasi et al., Astropart. Phys. 64, 49 (2015).

[7] W. Hanlon et al., arXiv:1310.0647.

[8] P. Abreu et al. (The Pierre Auger Collaboration), Astropart. Phys. 34, 314 (2010).

[9] R. Abbasi et al., Astrophys. J. Lett. 790, L21 (2014).

[10] R. Beck, AIP Conf. Proc. 1381, 117 (2011).

[11] P. Blasi and A. Olinto, Phys. Rev. D 59, 023001 (1998).

[12] D. Ryu, H. Kang, and P. Biermann, Astron. Astrophys. 335, 19 (1998).

[13] A. Abramowski et al., Astron. Astrophys. 562, A145 (2014).

[14] M. Ahlers, L. Anchordoqui, and A. Taylor, Phys. Rev. D 87, 023004 (2013).
[15] R. Tautz and A. Dosch, Phys. Plasmas 20, 022302 (2013).

[16] J. Giacalone and J. Jokipii, Astrophys. J. 520, 204 (1999).

[17] D. Harari et al., J. High Energy Phys. 0303 (2002) 045.

[18] N. Globus, D. Allard, and E. Parizot, Astron. Astrophys. 479, 97 (2008).

[19] D. Harari, S. Mollerach, and E. Roulet, Phys. Rev. D 89, 123001 (2014).

[20] B. Rouille d'Orfeuil et al., Astron. Astrophys. 567, A81 (2014).

[21] K. Kampert et al., Astopart. Phys. 42, 41 (2013).

[22] A. Mücke, R. Engel, J. Rachen, R. Protheroe, and T. Stanev, Comput. Phys. Commun. 124, 290 (2000).

[23] P. Abreu et al., J. Cosmol. Astropart. Phys. 05 (2013) 009.

[24] M. Ahlers et al., arXiv:1306.0910.

[25] A. Taylor, M. Ahlers, and F. Aharonian, Phys. Rev. D 84, 105007 (2011).

[26] Y. Takahashi and the JEM-EUSO Collaboration, New J. Phys. 11, 065009 (2009). 
[27] J. H. Adams, Jr. et al., Astropart. Phys. 44, 76 (2013).

[28] F. Salamida for the Pierre Auger Collaboration, arXiv:1107.4809 